\title{
Magneto-optical properties of single site-controlled InGaAsN quantum wires grown on prepatterned GaAs substrates
}

\author{
Marco Felici, ${ }^{1,2, *}$ Giorgio Pettinari, ${ }^{3,4}$ Romain Carron, ${ }^{2}$ Giovanna Lavenuta, ${ }^{1}$ Elena Tartaglini, ${ }^{1}$ Antonio Polimeni, ${ }^{1}$ \\ Dan Fekete, ${ }^{2,5}$ Pascal Gallo, ${ }^{2}$ Benjamin Dwir, ${ }^{2}$ Alok Rudra, ${ }^{2}$ Peter C. M. Christianen, ${ }^{3}$ Jan C. Maan, ${ }^{3}$ \\ Mario Capizzi, ${ }^{1}$ and Eli Kapon ${ }^{2}$ \\ ${ }^{1}$ Dipartimento di Fisica, Sapienza Università di Roma, P. le A. Moro 2, 00185 Roma, Italy \\ ${ }^{2}$ Laboratory of Physics of Nanostructures, Ecole Polytechnique Fédérale de Lausanne (EPFL), CH-1015 Lausanne, Switzerland \\ ${ }^{3}$ High Field Magnet Laboratory, Institute for Molecules and Materials, Radboud University Nijmegen, Toernooiveld 7 , \\ 6525 ED Nijmegen, The Netherlands \\ ${ }^{4}$ School of Physics and Astronomy, University of Nottingham, Nottingham NG7 2RD, United Kingdom \\ ${ }^{5}$ Department of Physics, Technion-Israel Institute of Technology, Haifa 32000, Israel
}

(Received 14 March 2012; published 23 April 2012)

\begin{abstract}
The properties of single site-controlled InGaAsN quantum wires (QWRs) — both untreated and irradiated with atomic hydrogen - are probed by micro-magnetophotoluminescence spectroscopy. The strong anisotropy of the diamagnetic shift measured for different orientations of the applied magnetic field confirms the one-dimensional nature of the QWR carrier wave function. In addition, the strain reduction associated with $\mathrm{N}$ incorporation is found to promote a larger indium intake in the QWR, enabling the realization of site-controlled QWRs emitting at long $(\geqslant 1.3 \mu \mathrm{m})$, technologically relevant wavelengths.
\end{abstract}

DOI: 10.1103/PhysRevB.85.155319

PACS number(s): 78.67.Lt, 62.23.St, 78.20.Ls, 81.07.Vb

\section{INTRODUCTION}

The quest for developing reliable methods for the growth of semiconductor nanostructures with controlled positions and finely tunable emission properties has quickly gained center stage in the emerging field of nanophotonics. ${ }^{1-7}$ The availability of such methods is indeed instrumental to the realization of novel devices-such as photonic crystal $(\mathrm{PhC})$ nanolasers $^{8-12}$ and highly efficient sources of single ${ }^{2,13}$ and entangled ${ }^{14}$ photons - wherein isolated quantum objects with well-defined confinement potentials need to be placed in spatial and spectral resonance with optical cavities of submicrometric dimensions.

In recent years, an effective technique for the fabrication of site-controlled homogeneous quantum wires (QWRs) naturally fit for integration with GaAs-based nanophotonic devices $^{8,15,16}$ - has been developed, based on metal-organic chemical vapor deposition (MOCVD) on (001) GaAs substrates patterned with ordered arrays of V grooves. ${ }^{17-21} \mathrm{Such} \mathrm{V}$ grooves are relatively long (up to several hundreds of microns) linear recesses, oriented along the [110] crystallographic direction and obtained through a combination of electron-beam lithography and wet chemical etching. ${ }^{15,18}$ Their distinctive $\mathrm{V}$-shaped cross section (typically having sub- $\mu \mathrm{m}$ width) is defined by two mirroring $\{111\} A$ crystal planes, exposed by the etching procedure. Due to a combination of growthrate anisotropy, curvature-induced capillarity, and entropy of mixing effects, ${ }^{21-23}$ MOCVD of a thin layer of InGaAs on a GaAs substrate patterned with $\mathrm{V}$ grooves results in the formation of a QWR along the main axis of each groove. Figure 1(a) displays a sketch of the grown QWR structure, characterized by a crescent-shaped cross section.

Very recently, ${ }^{24}$ this technique was successfully applied to the fabrication of high-quality InGaAsN/GaAs V-groove QWRs. Due to the unconventionally large band-gap shrinkage associated with $\mathrm{N}$ incorporation in III-V materials, ${ }^{25}$ a roomtemperature emission wavelength as high as $\sim 1.3 \mu \mathrm{m}$ was demonstrated for such QWRs, ${ }^{24}$ making them extremely attractive for the realization of compact light sources ${ }^{8,11}$ working in the wavelength window of interest for telecommunications. However, the structural and electronic properties of dilute-nitride V-groove QWRs need to be fully understood and characterized in order to exploit their considerable potential in the realization of advanced optoelectronic devices. With the present work, we take an important step in this direction by discussing and analyzing the results of micromagnetophotoluminescence measurements performed on single InGaAsN QWRs. Through the application of magnetic fields $(B)$ as high as $30 \mathrm{~T}$-and thanks to the possibility of applying $B$ with different orientations with respect to the QWRs ${ }^{19,26}$ - we were able to gather conclusive evidence of the presence of fully established two-dimensional confinement in the investigated nanostructures, as well as quantitative information on the QWR shape and composition. Particularly helpful, with regard to composition, was the possibility of measuring the magneto-optical properties of InGaAsN QWRs before and after hydrogen irradiation. Thanks to the wellknown capability of hydrogen to neutralize the effects of nitrogen in III-N-V alloys [such as GaAsN $,{ }^{27} \mathrm{InGaAsN},{ }^{28}$ and GaPN (Ref. 29)], indeed, $\mathrm{H}$ irradiation can be used as a tool to fine-tune the effective $\mathrm{N}$ concentration, thereby allowing for a precise assessment of the effects of $\mathrm{N}$ incorporation on a given sample.

\section{EXPERIMENT}

The investigated QWR samples were grown on semi-insulating (001) GaAs substrates, patterned with $1-\mu \mathrm{m}$-pitch arrays of [110]-oriented $\mathrm{V}$ grooves (groove width: $300 \mathrm{~nm}) .{ }^{24}$ Growth was performed in a low-pressure (20 mbar) MOCVD system, using trimethylGa/In as group-III precursors and $\mathrm{AsH}_{3}$ and 1,1-dimethylhydrazine for group V. The 3-nm-thick InGaAsN QWR layer (nominal In content 
(a)
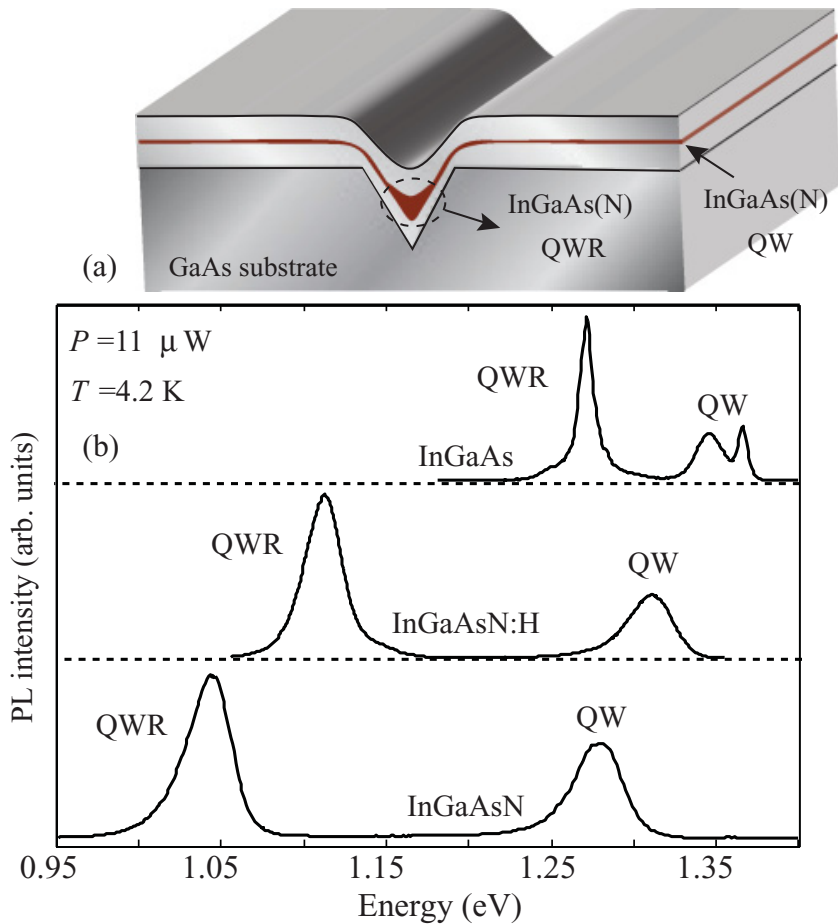

FIG. 1. (Color online) (a) Sketch of the QWR structure. (b) Micro-photoluminescence spectra $(T=4.2 \mathrm{~K}, P=11 \mu \mathrm{W}, B=$ $0 \mathrm{~T}$ ) of the investigated QWR samples (from bottom to top: untreated InGaAsN, fully hydrogenated InGaAsN, and InGaAs).

$[\mathrm{In}]=0.25)$ was grown with a two-step procedure aimed at ensuring a homogeneous $\mathrm{N}$ profile throughout the structure, ${ }^{30}$ and sandwiched between two 20-nm-thick GaAs layers [all quoted thicknesses are nominal and refer to growth on a planar (001) reference substrate].

Samples were mounted in a nonmagnetic Ti- and carbonbody insert, which was then placed in the bore of a $30 \mathrm{~T}$ watercooled Bitter magnet. The insert included a $40 \times$ microscope objective (with NA $=0.65$ and spatial resolution $\sim 1 \mu \mathrm{m}$, sufficient for performing single-QWR spectroscopy) and a three-axis piezoelectric nanopositioner. Two different sample holders, specifically designed for magnetophotoluminescence measurements with $B$ either parallel or perpendicular to the [001] crystallographic axis [i.e., to the direction of the collected photoluminescence (PL) signal], were alternatively employed. The PL signal was excited by a vanadate:Nd laser $(\lambda=532$ $\mathrm{nm}$ ), spectrally analyzed by a 0.30 -m monochromator (spectral resolution $=0.6 \mathrm{meV}$ ), and detected by a liquid $\mathrm{N}_{2}$-cooled InGaAs array. Throughout the measurements, the sample was kept at a temperature $T \sim 4.2 \mathrm{~K}$ in a liquid He-bath cryostat. For experiments requiring a higher sample temperature, a different cryostat was employed, which allowed for adjusting the temperature in the 10-290 K range (the spatial resolution was $\sim 20 \mu \mathrm{m}$ in this configuration). This option was selected, for example, when performing magneto-PL measurements on an InGaAs QWR sample, grown for reference purposes under the same conditions as the N-containing one (see Sec. III below). ${ }^{24}$ Indeed, the relatively low growth temperature $\left(520^{\circ} \mathrm{C}\right)$ set during the deposition of the QWR layer-chosen to maximize the $\mathrm{N}$ intake in dilute-nitride samples-resulted in the formation of a non-negligible concentration of localized states in the $\mathrm{N}$-free quantum wires. Hence, a sample temperature in the $10-40 \mathrm{~K}$ range was required when measuring InGaAs QWRs to minimize the effects of localized states on the PL spectra and to ensure the acquisition of reliable diamagnetic shift data.

Finally, $\mathrm{H}$ irradiation was performed at $T=300{ }^{\circ} \mathrm{C}$ with a low-energy $(\sim 100 \mathrm{eV})$ ion gun. $\mathrm{N}$ passivation of the InGaAsN QWR samples was deemed complete as soon as the QWR emission peak stopped blueshifting for an increasing $\mathrm{H}$ dose [ $=6 \times 10^{16} \mathrm{H}^{+}$ions $/ \mathrm{cm}^{2}$ for the sample displayed in Fig. 1(b)].

\section{RESULTS AND DISCUSSION}

\section{A. Zero magnetic field $(B=0 \mathrm{~T})$}

Figure 1(b) shows representative micro-PL spectra (acquired at $B=0 \mathrm{~T}$ ) of the three samples studied in the present work. The two spectra at the bottom and in the center of the figure refer to the same InGaAsN QWR sample before and after hydrogen irradiation, respectively. The topmost spectrum is associated with a reference InGaAs QWR sample, grown under the same conditions as the $\mathrm{N}$-containing one. ${ }^{24}$ In each spectrum, the lower-energy peak is associated with the groundstate transition from the QWR, while higher-energy features are related to the quantum well $(\mathrm{QW})$ grown on the planar part of the (001) GaAs substrate [see Fig. 1(a)]. It is interesting to note that in the reference InGaAs sample, the localized states associated with the relatively low growth temperature of the QWR layer $\left(520^{\circ} \mathrm{C}\right)$ give rise to a second QW-related peak (the broader, lower-energy one), which quickly disappears with increasing temperature.

The passivation of $\mathrm{N}$ in the hydrogenated InGaAsN sample is evidenced by the 70 and $\sim 30 \mathrm{meV}$ blueshifts, respectively, observed for the QWR and planar-QW peaks upon hydrogen irradiation. The emission energies of the fully passivated QWR $(1.11 \mathrm{eV})$ and $\mathrm{QW}(1.31 \mathrm{eV})$ are consistent with an indium content of at least $\sim 0.4$ and $\sim 0.2-0.25$, respectively ${ }^{31}$ while the magnitude of the $\mathrm{H}$-induced blueshifts suggests initial $\mathrm{N}$ concentrations $(x)$ approximately equal to $1 \%$ (for the QWR) and $0.5 \%$ (for the QW). These estimates are based on the results reported in Ref. 28, wherein shifts of $\sim 50$ and $\sim 140 \mathrm{meV}$ were measured for $\operatorname{In}_{y} \mathrm{Ga}_{1-y} \mathrm{As}_{1-x} \mathrm{~N}_{x} \mathrm{QW}$ samples with $x=0.7 \%(y=34 \%)$ and $x=2.2 \%(y$ $=41 \%$ ). As we will see in the following, the value of $x$ estimated for the InGaAsN QWRs is also compatible with the electron effective mass obtained through the analysis of the magneto-PL data. However, this value only amounts to roughly one-half of the estimate- $x=2.1 \%$-obtained in Ref. 24 by fitting the temperature dependence of the QWR micro-PL peak. The origin of such a large discrepancy lies primarily in the assumption - made throughout Ref. 24-of a constant In content $([\mathrm{In}]=0.25)$ in the $\operatorname{InGaAs}(\mathrm{N})$ QWRs, regardless of whether they are N-containing or not. As we just discussed, however, the emission energy of the fully passivated InGaAsN QWR seems to suggest an indium content of no less than $\sim 0.4$. This is in stark contrast with the observations made in the InGaAs reference sample, for which both the QWR emission energy $(1.27 \mathrm{eV})$ and energy-dispersive x-ray spectroscopy measurements ${ }^{32}$ indeed confirm an indium content 
of $\sim 0.25-0.3$ in the QWR. As a first step toward an explanation for the seemingly much higher In intake in the InGaAsN QWRs, we would like to note that the equilibrium In concentration in V-groove InGaAs QWRs is the result of a balance between two competing mechanisms. On the one hand, the higher diffusion coefficient of indium with respect to gallium enhances the capillarity-induced flux of In adatoms toward the bottom of the groove, yielding a higher-than-nominal In concentration in the QWR. On the other hand, the presence of strain in the $\operatorname{InGaAs}(\mathrm{N})$ layer sets a limit for the maximum lattice mismatch (i.e., the maximum In content) that can be accommodated in the QWR region. The introduction of macroscopic concentrations of nitrogen in the QWR layer results in a considerable strain reduction $\left[\mathrm{In}_{y} \mathrm{Ga}_{1-y} \mathrm{As}_{1-x} \mathrm{~N}_{x}\right.$ is lattice-matched to GaAs for $y \sim 3 x$ (Ref. 33)], and is therefore likely to play a major role in the increase of the indium intake observed in InGaAsN QWRs.

\section{B. Measurements with varying magnetic field $(B \neq 0 \mathrm{~T})$}

Before moving on to the discussion of the magneto-PL measurements performed on the $\operatorname{InGaAs}(\mathrm{N})$ QWRs described above, we briefly outline the theoretical framework in which our experimental results are analyzed. The key features of the response to an applied magnetic field of the carriers (electrons or holes) confined in a V-groove QWR can be effectively reproduced by approximating the QWR in terms of a two-dimensional anisotropic parabolic potential. ${ }^{26}$ Excitonic effects, while not explicitly included in the model, are effectively accounted for by the parabolic potential. ${ }^{34}$ The diamagnetic shift, $\Delta E_{d, j}$, of the QWR peak in the presence of a magnetic field oriented along a generic direction $j$ can then be written as

$$
\begin{aligned}
\Delta E_{d, j}= & \frac{1}{2} \hbar \sum_{p=e, h}\left[\sqrt{\left[\omega_{c}^{p, j}(B)\right]^{2}+\left(\sum_{k \neq j} \Omega_{\mathrm{QWR}}^{p, k}\right)^{2}}\right. \\
& \left.-\sum_{k \neq j} \Omega_{\mathrm{QWR}}^{p, k}\right] .
\end{aligned}
$$

Here, $\omega_{c}^{p, j}(B)=q B / m_{\perp, p}^{j}$ (where $p$ identifies either electrons, $e$, or holes, $h$, and $m_{\perp, p}^{j}$ is the average carrier mass in the plane perpendicular to $B)$ is the cyclotron frequency for $B$ oriented in the direction $j[j=[001],[110]$, or [110]; see Fig. 2(a)], while $\Omega_{\mathrm{OWR}}^{p, k}$ is the oscillator frequency of the parabolic potential in the direction $k$. In order to extract an effective QWR "size" along the different confinement directions, $\frac{1}{2} \hbar \Omega_{\mathrm{QWR}}^{p, k}$ was set equal to $(\hbar \pi)^{2} / 2 m_{p}^{k} L_{k}^{2}$, namely, the confinement energy of a particle with mass $m_{p}^{k}$ in an infinite potential well of width $L_{k}$.

In InGaAs and hydrogenated InGaAsN QWRs, the hole masses in the [001] and [110] directions-as well as the (isotropic) electron effective mass-depend only on [In] (respectively fixed at 0.25 and 0.4 for InGaAs and InGaAsNboth hydrogenated and not-QWRs), and can thus be evaluated via simple interpolations. ${ }^{31}$ Along the QWR main axis

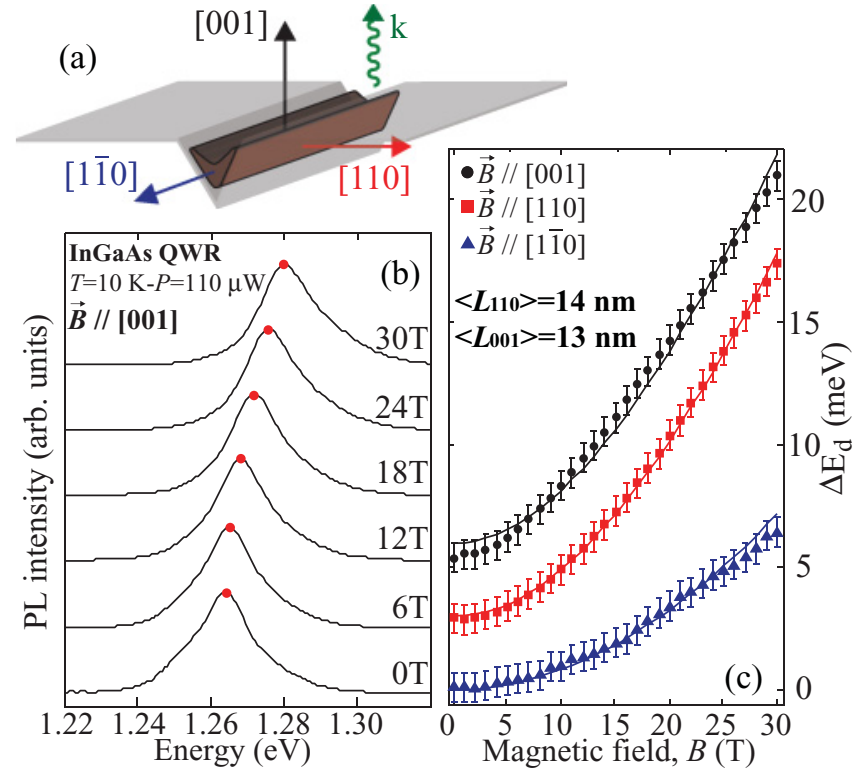

FIG. 2. (Color online) (a) Sketch of the possible orientations of $B$ with respect to the QWR employed during the measurements (indicated by red, blue, and black arrows, respectively, parallel to the [110], [110], and [001] crystallographic axes). The (green) zigzag arrow indicates the direction of the wave-number vector $(\mathbf{k})$ of the collected PL signal for all the configurations considered. (b) PL spectra $(T=10 \mathrm{~K}, P=110 \mu \mathrm{W})$ of an InGaAs QWR array, taken at different values of $B(B \|[001])$. The red dots correspond to the peak position, as obtained through a fitting procedure. (c) Diamagnetic shift of the InGaAs QWR peak for three different configurations (different datasets were vertically shifted for clarity): $B \|$ [001] (black dots), $B \|$ [110] (red squares), and $B \|[1 \overline{1} 0]$ (blue triangles). The solid lines were obtained by fitting Eq. (1) to the experimental data. The fit gave estimates of $L_{110}=(14.0 \pm 2.5) \mathrm{nm}$ and of $L_{001}=13$ $\mathrm{nm}$. Carrier masses were fixed to $m_{e}=0.055 m_{0}, m_{h}^{[001]}=0.37 m_{0}$, $m_{h}^{[110]}=0.63 m_{0}$, and $m_{h}^{[110]}=0.12 m_{0}$ [corresponding to [In] $=0.25$ (Ref. 31)].

(in the [110] direction), on the other hand, we can use the value $m_{h}^{[1 \overline{1} 0]}=0.12 m_{0}$ (where $m_{0}$ is the free-electron mass), measured in Ref. 35 in the plane of a (001) InGaAs QW (for a discussion of the effects of confinement on the hole mass in semiconductor heterostructures, see Ref. 36). When applying Eq. (1) to N-free and fully hydrogenated QWRs, then, the only unknown parameters are the effective wire thicknesses along the directions of confinement, $L_{110}$ and $L_{001}$ (the confinement length along the main axis of the QWR, $L_{1 \overline{1} 0}$, can be safely set to $+\infty$ ). These effective thicknesses can be estimated by separately fitting Eq. (1) to the diamagnetic shifts measured with $B$ parallel to the [001] and [110] directions, respectively. As summarized in Fig. 2, the application of this procedure to the InGaAs QWR sample investigated in this work yields-after averaging over values extracted from several different datasets $-L_{110}=(14.0 \pm 2.5) \mathrm{nm}$ and $L_{001}=$ $13 \mathrm{~nm} .{ }^{37}$ The reliability of these values can be independently tested by inserting them in the formula of the diamagnetic shift for $B$ applied along the QWR ([11 0$\rangle$ direction, blue triangles in the figure). As shown in Fig. 2(c), the agreement between theory and experiments is quite remarkable. 


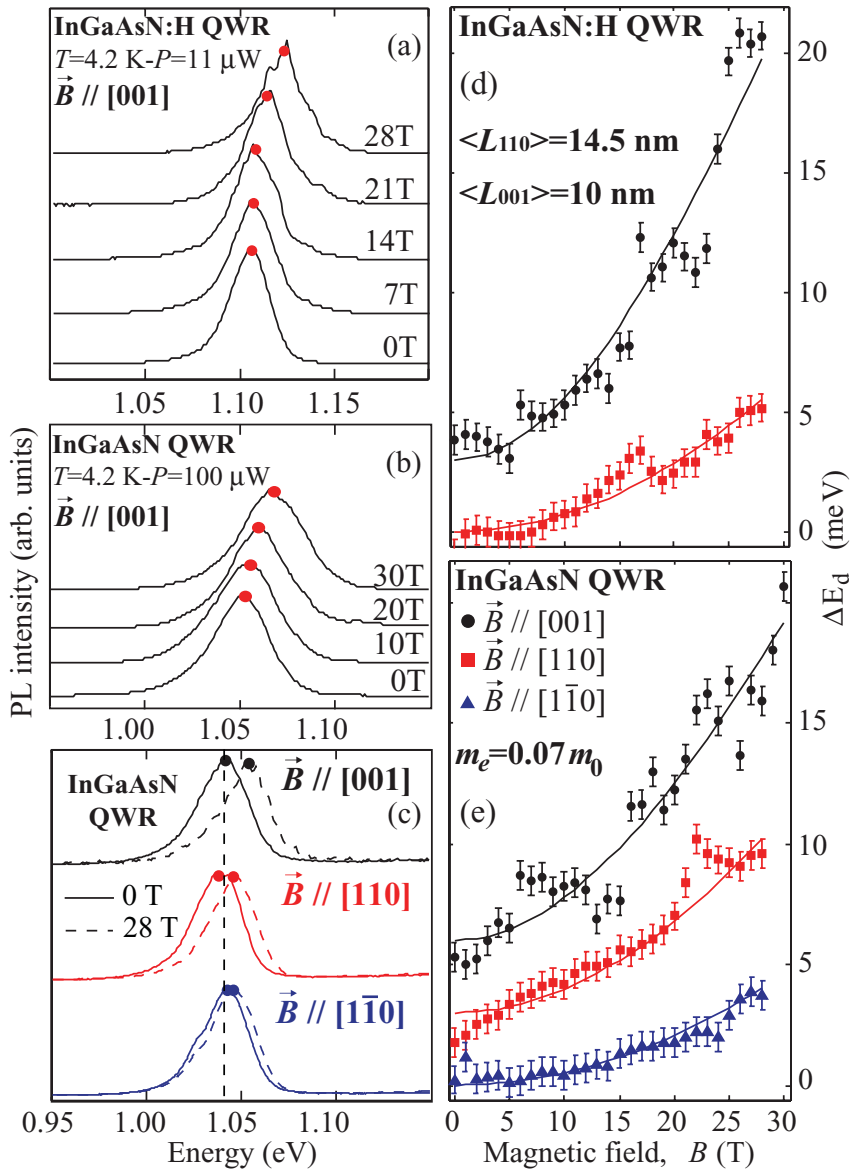

FIG. 3. (Color online) (a) Micro-PL spectra $(T=4.2 \mathrm{~K}, P=11$ $\mu \mathrm{W})$ of a single hydrogenated InGaAsN QWR for different values of $B(B \|[001])$. The red dots correspond to the peak position, as obtained through a fitting procedure. (b) Same as in (a), but for an untreated InGaAsN QWR $(T=4.2 \mathrm{~K}, P=100 \mu \mathrm{W})$. (c) Comparison between the zero- (solid line) and high-field $(B=28$ $\mathrm{T}$, dashed line) micro-PL spectra of an untreated InGaAsN QWR, taken with $B \|$ [001] (black), $B \|$ [110] (red), and $B \|$ [110] (blue). (d) Diamagnetic shift of the hydrogenated InGaAsN QWR peak, for $B \|$ [001] (black dots) and $B \|$ [110] (red squares). (e) Diamagnetic shift of the untreated InGaAsN QWR peak, for $B \|$ [001] (black dots), $B \|[110]$ (red squares), and $B \|[1 \overline{1} 0]$ (blue triangles). In (d) and (e), different datasets were vertically shifted for clarity; the solid lines were obtained by fitting Eq. (1) to the experimental data. The fits relative to the hydrogenated QWR yielded estimates of $L_{110}=$ $(14.5 \pm 1.5) \mathrm{nm}$ and of $L_{001}=(10.0 \pm 2.0) \mathrm{nm}$ [carrier masses were fixed to $m_{e}=0.048 m_{0}, m_{h}^{[001]}=0.38 m_{0}, m_{h}^{[110]}=0.62 m_{0}$, and $m_{h}^{[1 \overline{1} 0]}$ $=0.12 m_{0}$, corresponding to $[\mathrm{In}]=0.4$ (Ref. 31) . The fits relative to the untreated InGaAsN QWR were then performed with $m_{e}$ as the only free parameter, obtaining $m_{e}=(0.07 \pm 0.01) m_{0}$.

The first step of the magneto-optical characterization of single N-containing QWRs-whose main results are summarized in Fig. 3-also consisted in the determination of the wire thicknesses along the [110] and [001] directions. This procedure was performed on the fully hydrogenated sample, wherein the additional degree of freedom associated with the presence of nitrogen in the material is effectively "frozen" because of the formation of N-H complexes upon $\mathrm{H}$ irradiation. ${ }^{28}$ After fixing the effective masses of carriers to their InGaAs $([\mathrm{In}]=0.4)$ values, the average $L_{110}$ and $L_{001}$ were determined to be equal to $(14.5 \pm 1.5)$ and $(10.0 \pm 2.0)$ $\mathrm{nm}$, respectively. The value obtained for $L_{110}$ is nearly identical to the one estimated for the InGaAs QWRs, and is consistent with the commonly held view that the lateral width of V-groove QWRs is primarily determined by the radius of curvature the groove bottom has when the QWR layer is deposited ( $\sim 10$ $20 \mathrm{~nm}$ according to Ref. 22). On the other hand, the estimated QWR thickness $\left(L_{001}=10 \mathrm{~nm}\right)$ closely matches the value $(10.5 \mathrm{~nm})$ determined by transmission electron microscopy (TEM). ${ }^{24}$ Interestingly, TEM measurements ${ }^{32}$ also suggest a slightly larger wire thickness for the (N-free) InGaAs QWR sample, in agreement with the value of $L_{001}=13 \mathrm{~nm}$ estimated here from diamagnetic shift data.

The estimates of $L_{110}$ and $L_{001}$ attained on the hydrogenated sample were then inserted as constants in Eq. (1), and the latter-with the electron mass, $m_{e}$, as the only free parameter-was fitted to the measured diamagnetic shifts of the untreated InGaAsN QWRs. The fitting procedure was applied to datasets acquired with $B$ parallel to the [001] and [110] directions, yielding an average $m_{e}=(0.07 \pm 0.01) m_{0}$. The values of $L_{110}, L_{001}$, and $m_{e}$ estimated with this method were then used to compute the diamagnetic shift expected for $B$ parallel to the QWR, obtaining the (blue) curve displayed in the lowest part of Fig. 3(e). The good agreement observed between this curve and the experimental data [blue triangles in Fig. 3(e)] further supports the validity of our approach. Moreover, the estimated value of the electron effective mass is compatible ${ }^{38}$ with a nitrogen concentration of $\sim 1 \%$, which was also inferred from the $\mathrm{H}$-induced blueshift of the QWR PL peak displayed in Fig. 1(b). ${ }^{28}$ It is interesting to note that even though the estimates of $L_{110}, L_{001}$, and $m_{e}$ (and thus of $x$ ) are-in principle-dependent on the value chosen for the In content in the wire (set to $[\mathrm{In}]=0.4-$ based on the emission energy of the fully hydrogenated InGaAsN QWR - in the analysis described above), in practice this dependence is relatively weak. Indeed, if [In] is varied between 0.25 (the nominal In content of the deposited InGaAsN layer) and 0.55 , the values of $m_{e}$ and of the effective QWR thicknesses change by less than the experimental uncertainty given by QWR-to-QWR fluctuations (equal to $\pm 0.01 m_{0}, \pm 1.5 \mathrm{~nm}$, and $\pm 2.0 \mathrm{~nm}$ for $m_{e}, L_{110}$, and $L_{001}$, respectively).

\section{CONCLUSIONS}

In summary, we performed an in-depth magneto-optical characterization of single dilute nitride-based quantum wires, emitting at $1.3 \mu \mathrm{m}$ at room temperature. The application of high magnetic fields with varying orientation with respect to the QWR allowed us to confirm the one-dimensional character of the wave function of carriers localized in the QWR. Further, we could estimate the effective wire thicknesses along different confinement directions, finding values in substantial agreement with those measured by TEM. In addition, the possibility to "turn off" the effects of $\mathrm{N}$ incorporation by hydrogen irradiation enabled us to correct previous estimates ${ }^{24}$ of the concentration of $\mathrm{N}$ (and In) in the QWR. The relatively moderate amount of $\mathrm{N}(x \sim 1 \%)$ needed to reach an emission wavelength of $1.3 \mu \mathrm{m}$-seemingly due to the role played by nitrogen in increasing the In intake in the QWR - suggests the 
potential for obtaining dilute-nitride QWRs emitting at much longer wavelengths $(1.55 \mu \mathrm{m}$ and beyond), while keeping the $\mathrm{N}$ concentration below the threshold that ensures a good optical quality of the grown material $(x<4 \%-5 \%){ }^{39}$

In perspective, the inherent site control of InGaAsN-based V-groove QWRs makes them naturally suited for the integration with advanced nanophotonic devices, and particularly with new-generation, high-quality factor microcavities based on modified $\mathrm{PhC}$ waveguides. ${ }^{40-42}$ Moreover, the possibility of fine-tuning the effective $\mathrm{N}$ concentration in different regions of the QWR by spatially selective hydrogenation ${ }^{7,43,44}$ opens new avenues to the post-growth engineering of the confinement potential of single semiconductor nanostructures.

\section{ACKNOWLEDGMENTS}

Part of this work has been supported by EuroMagNET II under EU Contract No. 228043. G.P. would like to acknowledge funding by the EU under grant agreement No. PIEF-GA-2010-272612. We also wish to acknowledge support from COST Action MP0805, and funding from the Swiss National Science Foundation. *marco.felici@roma1.infn.it

${ }^{1}$ S. Kiravittaya, A. Rastelli, and O. Schmidt, Appl. Phys. Lett. 88, 043112 (2006).

${ }^{2}$ A. Mohan, P. Gallo, M. Felici, B. Dwir, A. Rudra, J. Faist, and E. Kapon, Small 6, 1268 (2010).

${ }^{3}$ A. Huggenberger, S. Heckelmann, C. Schneider, S. Hoefling, S. Reitzenstein, L. Worschech, M. Kamp, and A. Forchel, Appl. Phys. Lett. 98, 131104 (2011).

${ }^{4}$ S. Hughes, P. Yao, F. Milde, A. Knorr, D. Dalacu, K. Mnaymneh, V. Sazonova, P. J. Poole, G. C. Aers, J. Lapointe, R. Cheriton, and R. L. Williams, Phys. Rev. B 83, 165313 (2011).

${ }^{5}$ J. Skiba-Szymanska, A. Jamil, I. Farrer, M. B. Ward, C. A. Nicoll, D. J. P. Ellis, J. P. Griffiths, D. Anderson, G. A. C. Jones, D. A. Ritchie, and A. J. Shields, Nanotechnology 22, 065302 (2011).

${ }^{6}$ J. Yuan, H. Wang, R. P. J. Van Veldhoven, J. Wang, T. De Vries, B. Smalbrugge, C. Y. Jin, P. Nouwens, E. J. Geluk, A. Y. Silov, and R. Noetzel, Appl. Phys. Lett. 98, 201904 (2011).

${ }^{7}$ R. Trotta, A. Polimeni, F. Martelli, G. Pettinari, M. Capizzi, L. Felisari, S. Rubini, M. Francardi, A. Gerardino, P. C. M. Christianen, and J. C. Maan, Adv. Mater. 23, 2706 (2011).

${ }^{8}$ K. A. Atlasov, M. Calic, K. F. Karlsson, P. Gallo, A. Rudra, B. Dwir, and E. Kapon, Opt. Express 17, 18178 (2009).

${ }^{9}$ O. Painter, R. K. Lee, A. Scherer, A. Yariv, J. D. O'Brien, P. D. Dapkus, and I. Kim, Science 284, 1819 (1999).

${ }^{10}$ S. Strauf, K. Hennessy, M. T. Rakher, Y. S. Choi, A. Badolato, L. C. Andreani, E. L. Hu, P. M. Petroff, and D. Bouwmeester, Phys. Rev. Lett. 96, 127404 (2006).

${ }^{11}$ K. A. Atlasov, A. Rudra, B. Dwir, and E. Kapon, Opt. Express 19, 2619 (2011).

${ }^{12}$ M. Nomura, N. Kumagai, S. Iwamoto, Y. Ota, and Y. Arakawa, Nat. Phys. 6, 279 (2010).

${ }^{13}$ M. Calic, P. Gallo, M. Felici, K. A. Atlasov, B. Dwir, A. Rudra, G. Biasiol, L. Sorba, G. Tarel, V. Savona, and E. Kapon, Phys. Rev. Lett. 106, 227402 (2011).

${ }^{14}$ A. Mohan, M. Felici, P. Gallo, B. Dwir, A. Rudra, J. Faist, and E. Kapon, Nat. Photon. 4, 302 (2010).

${ }^{15}$ K. A. Atlasov, K. F. Karlsson, E. Deichsel, A. Rudra, B. Dwir, and E. Kapon, Appl. Phys. Lett. 90, 153107 (2007).

${ }^{16}$ K. A. Atlasov, M. Felici, K. F. Karlsson, P. Gallo, A. Rudra, B. Dwir, and E. Kapon, Opt. Express 18, 117 (2010).

${ }^{17}$ E. Kapon, D. M. Hwang, and R. Bhat, Phys. Rev. Lett. 63, 430 (1989).

${ }^{18}$ E. Colas, S. Simhony, E. Kapon, R. Bhat, D. Hwang, and P. Lin, Appl. Phys. Lett. 57, 914 (1990).
${ }^{19}$ Y. Nagamune, Y. Arakawa, S. Tsukamoto, M. Nishioka, S. Sasaki, and N. Miura, Phys. Rev. Lett. 69, 2963 (1992).

${ }^{20}$ F. Vouilloz, D. Y. Oberli, M. A. Dupertuis, A. Gustafsson, F. Reinhardt, and E. Kapon, Phys. Rev. Lett. 78, 1580 (1997).

${ }^{21}$ G. Biasiol and E. Kapon, Phys. Rev. Lett. 81, 2962 (1998).

${ }^{22}$ G. Biasiol, A. Gustafsson, K. Leifer, and E. Kapon, Phys. Rev. B 65, 205306 (2002).

${ }^{23}$ E. Pelucchi, V. Dimastrodonato, A. Rudra, K. Leifer, E. Kapon, L. Bethke, P. A. Zestanakis, and D. D. Vvedensky, Phys. Rev. B 83, 205409 (2011).

${ }^{24}$ R. Carron, D. Fekete, P. Gallo, B. Dwir, A. Rudra, M. Felici, B. Bartova, M. Cantoni, and E. Kapon, Appl. Phys. Lett. 99, 101107 (2011).

${ }^{25}$ E. P. O'Reilly, A. Lindsay, P. J. Klar, A. Polimeni, and M. Capizzi, Semicond. Sci. Technol. 24, 033001 (2009).

${ }^{26}$ T. Tanaka, Y. Arakawa, and G. W. E. Bauer, Phys. Rev. B 50, 7719 (1994).

${ }^{27}$ M. Felici, R. Trotta, F. Masia, A. Polimeni, A. Miriametro, M. Capizzi, P. J. Klar, and W. Stolz, Phys. Rev. B 74, 085203 (2006).

${ }^{28}$ A. Polimeni, G. Baldassarri, H. Bissiri, M. Capizzi, M. Fischer, M. Reinhardt, and A. Forchel, Phys. Rev. B 63, 201304 (2001).

${ }^{29}$ A. Polimeni, M. Bissiri, M. Felici, M. Capizzi, I. A. Buyanova, W. M. Chen, H. P. Xin, and C. W. Tu, Phys. Rev. B 67, 201303 (2003).

${ }^{30}$ D. Fekete, R. Carron, P. Gallo, B. Dwir, A. Rudra, and E. Kapon, Appl. Phys. Lett. 99, 072116 (2011).

${ }^{31}$ I. Vurgaftman, J. R. Meyer, and L. R. Ram-Mohan, J. Appl. Phys. 89, 5815 (2001).

${ }^{32}$ B. Bartova and M. Cantoni (private communication).

${ }^{33}$ C. H. Fischer and P. Bhattacharya, J. Appl. Phys. 96, 4176 (2004).

${ }^{34}$ In general, excitonic effects are responsible for a slowdown of the diamagnetic shift, which in turn results in an increase of the carrier masses and/or a decrease of the effective QWR thicknesses estimated by fitting Eq. (1) to the experimental data. In Ref. 26, however, this slowdown was estimated to be less than $10 \%$ of the total diamagnetic shift, or $\sim 1 \mathrm{meV}$ at $30 \mathrm{~T}$.

${ }^{35}$ T. Wimbauer, K. Oettinger, A. L. Efros, B. K. Meyer, and H. Brugger, Phys. Rev. B 50, 8889 (1994).

${ }^{36}$ P. Y. Yu and M. Cardona, Fundamentals of Semiconductors: Physics and Materials Properties (Springer, Berlin, 2001), pp. 483-487.

${ }^{37}$ Only one dataset was acquired in this configuration, making it impossible to define a standard deviation. 
${ }^{38}$ G. B. von Högersthal, A. Polimeni, F. Masia, M. Bissiri, M. Capizzi, D. Gollub, M. Fischer, and A. Forchel, Phys. Rev. B 67, 233304 (2003).

${ }^{39}$ H. Y. Liu, M. Hopkinson, P. Navaretti, M. Gutierrez, J. S. Ng, and J. P. R. David, Appl. Phys. Lett. 83, 4951 (2003).

${ }^{40}$ B. Song, S. Noda, T. Asano, and Y. Akahane, Nat. Mater. 4, 207 (2005).

${ }^{41}$ E. Kuramochi, M. Notomi, S. Mitsugi, A. Shinya, T. Tanabe, and T. Watanabe, Appl. Phys. Lett. 88, 041112 (2006).
${ }^{42}$ M. Felici, K. A. Atlasov, A. Surrente, and E. Kapon, Phys. Rev. B 82, 115118 (2010)

${ }^{43}$ M. Felici, A. Polimeni, G. Salviati, L. Lazzarini, N. Armani, F. Masia, M. Capizzi, F. Martelli, M. Lazzarino, G. Bais, M. Piccin, S. Rubini, and A. Franciosi, Adv. Mater. 18, 1993 (2006).

${ }^{44}$ R. Trotta, A. Polimeni, M. Capizzi, F. Martelli, S. Rubini, M. Francardi, A. Gerardino, and L. Mariucci, Appl. Phys. Lett. 94, 261905 (2009). 